\title{
ON THE REIDEMEISTER MOVES \\ OF A CLASSICAL KNOT
}

\author{
BRUCE TRACE
}

\begin{abstract}
We show that under certain reasonable criteria one of the Reidemeister moves can be elminated.
\end{abstract}

It is a well-known fact in classical knot theory that the following three moves, known as the Reidemeister moves, generate all the possible projections of a knot. To be more precise, given two oriented knots, $K_{1}$ and $K_{2}$, in $\mathbf{R}^{3}$, where $K_{i}$ is in general position with respect to the natural projection $\pi: \mathbf{R}^{3} \rightarrow \mathbf{R}^{2}$, having the property that there is an isotopy carrying $K_{1}$ to $K_{2}$ and preserving the orientations of the knots, then there is a sequence of the Reidemeister moves taking $K_{1}$ to $K_{2}$ and preserving the orientations of the knots. Note that the last two Reidemeister moves preserve two properties of the knots projection.

(1)

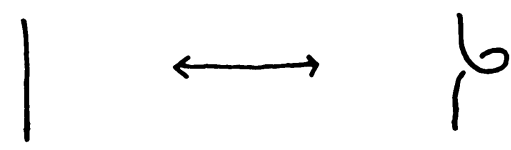

(2)

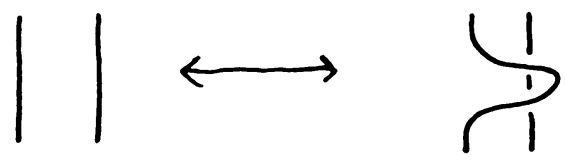

(3)
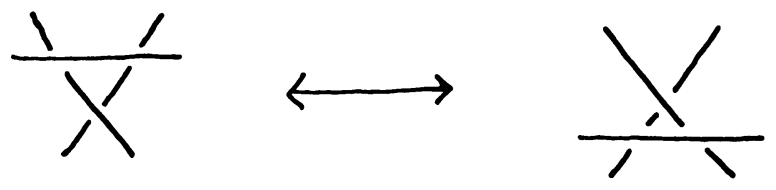

FIGURE 1. The Reidemeister moves

The first is the winding number, the second is the framing associated to the knot's projection. Upon defining these concepts we will show that given two projections of an oriented knot having the same winding numbers and associated framings then only the last two Reidemeister moves are required in passing from either of the projections to the other.

Received by the editors January 24, 1983. Presented to the AMS at the Norman, Oklahoma, meeting, March 1983.

1980 Mathematics Subject Classification. Primary 57C99, 57065.

${ }^{1}$ Research supported in part by NSF Grant MCS-810-3387. 
Suppose $f: S^{1} \rightarrow \mathbf{R}^{3}$ is a smooth embedding. Assume that $f$ is in general position with respect to $\pi$, i.e., the singular set of $f$ consists of a finite number of transverse double points and $\pi \circ f: S^{1} \rightarrow \mathbf{R}^{2}$ is an immersion. We endow $S^{1}$ with its usual counterclockwise orientation. Let $\mathbf{X}$ denote a nonzero vector field on $S^{1}$ inducing this orientation. Then since $\pi \circ f$ is an immersion, $(\pi \circ f)_{*} \mathbf{X} \neq 0$, so we obtain a map, $S^{1} \rightarrow S^{1}$, the degree of which is called the winding number of $f$. This induced map is usually called the Gauss map. To define the framing associated to $f$ we position ourselves high on the $z$-axis. From this vantage point the "double points" of $f\left(S^{1}\right)$ fall naturally into two types, $\uparrow$ and $\uparrow$. We assign $\mathrm{a}-1$ to $\uparrow$ and a +1 to $\uparrow$. The sum of these \pm 1 's is the framing associated to $f$.

We are now in a position to prove the

TheOREM. Suppose $f, g: S^{1} \rightarrow \mathbf{R}^{3}$ are general position embeddings representing the same oriented knot such that the winding number of $f$ equals the winding number of $g$ and the associated framing of $f$ equals the associated framing of $g$. Then we can pass from $f\left(S^{1}\right)$ to $g\left(S^{1}\right)$ via the second and third Reidemeister moves.

Proof. We begin by viewing $f\left(S^{1}\right)$ in $\mathbf{R}^{3} \times\{0\}$ and $g\left(S^{1}\right)$ in $\mathbf{R}^{3} \times\{1\}$. Since $f\left(S^{1}\right)$ and $g\left(S^{1}\right)$ represent the same oriented knot we can pass from $f\left(S^{1}\right)$ to $g\left(S^{1}\right)$ via the Reidemeister moves preserving the knot's orientation. Beginning at $f\left(S^{1}\right)$ we build an isotopy in $\mathbf{R}^{3} \times I$ as follows: whenever a Reidemeister move of the second or third type is required we perform the operation. For changes involving the first Reidemeister move there are two basic cases: whenever the introduction of a kink is required we do so-but we also introduce a kink at each previous level in the isotopy already constructed including the $\left(\mathbf{R}^{3} \times 0\right)$-level, if we must eliminate a kink we proceed as in Figure 2.

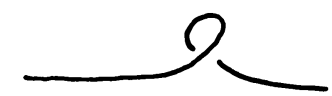

kink to be eliminated

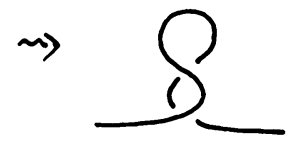

perform an isotopy
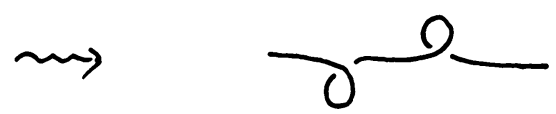

introduce kink and run introduced kink back down the isotopy

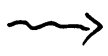

Figure 2. EliminatiNg KINKS

Proceeding in this fashion, we end up with an isotopy joining $f\left(S^{1}\right)+$ (kinks) to $g\left(S^{1}\right)$ where this isotopy involves only the second and third Reidemeister moves.

We can assume that all the kinks added to $f\left(S^{1}\right)$ lie on a subarc of $f\left(S^{1}\right)$ having no double points i.e. we have a picture looking like sesr. The previous picture contains the four possible types of kinks which can occur. Kinks lying above the 
subarc will change the winding number of $f$ by either +1 or -1 depending on orientation, those below would change the winding number of $f$ by -1 or +1 , respectively, depending on orientation. (The important relationship is +1 above then -1 below, or -1 above then +1 below.) The framing associated to each kink then provides the four possibilities. Now let $a_{+}$denote the number of +1 framed kinks lying above the subarc, $a_{-}$denote the number of -1 framed kinks lying above the subarc and $b_{+}, b_{-}$denote the corresponding numbers for kinks below the subarc.

Since the winding number of $f$ equals the winding number $g$, and type 2 and 3 Reidemeister moves do not alter the winding number we must have $a_{+}+a_{-}=b_{+}$ $+b_{-}$. An analogous statement applied to associated framing yields $a_{+}+b_{+}=a_{-}+b_{-}$. One easily verifies that for these equations to hold simultaneously we must have $a_{+}=b_{-}$and $a_{-}=b_{+}$. These are precisely the equations required to apply the argument used for eliminating kinks $\left(a_{+}+a_{-}\right)$-times yielding $f\left(S^{1}\right)+($ kinks $)$ can be altered to $f\left(S^{1}\right)$ via the Reidemeister moves of the second and third types. This completes the proof of the Theorem.

We complete this paper with some remarks. Note that every oriented knot has a projection with winding number $=0$ and framing $=1$. (In general, (winding number $)+($ framing $)=($ odd number $)$.) The quickest approach to observing this remark is to take a braid presentation for the knot and introduce kinks to arrive at the desired winding number and framing. The usefulness of winding number $=0$ and framing $=1$ projections is that they are invariant under change of the knots orientation. (In general, changing orientation multiplies winding number by -1 and leaves framing fixed.) These observations yield

COROllary 1. Let $K_{1}$ and $K_{2}$ denote two knots with winding number $=0$ and framing $=1$. Then $K_{1}$ is isotopic to $K_{2}$ (as unoriented knots) if and only if we can pass from $K_{1}$ to $K_{2}$ using only the second and third Reidemeister moves.

Lastly, we note that the Theorem is a strenthened version of the Whitney-Graustein Theorem, i.e.

COROllary 2 (Whitney-Graustein TheOREM). Regular homotopy classes of immersions $S^{1} \rightarrow \mathbf{R}^{2}$ are in one-to-one correspondence with $\mathbf{Z}$, the bijection being induced by the Gauss map.

The key to observing this is that every immersion $S^{1} \rightarrow \mathbf{R}^{2}$ may be viewed as the projection of the unknot into $\mathbf{R}^{2}$ and the unknot with standard orientation is isotopic to the unknot with opposite orientation.

\section{REFERENCES}

[G] C. Gordon, Some aspects of knot theory, Lecture Notes in Math., vol. 685, Springer-Verlag, New York, 1978.

Department of Mathematics, University of Utah, Salt Lake City, Utah 84112

Current address: Department of Mathematics, Southwest Texas State University, San Marcos, Texas 78666 